\title{
Stabilization with Optimal Performance for Dissipative Discrete-Time Impulsive Hybrid Systems
}

\author{
Lamei Yan ${ }^{1}$ and Bin Liu²,3 \\ ${ }^{1}$ School of Printing Engineering, Hangzhou Dianzi University, Hangzhou 310018, China \\ ${ }^{2}$ Department of Information Engineering, The Australian National University, ACT 0200, Australia \\ ${ }^{3}$ College of Science, Hunan University of Technology, Zhuzhou 412008, China
}

Correspondence should be addressed to Bin Liu, bin.liu@anu.edu.au

Received 14 September 2009; Accepted 16 April 2010

Academic Editor: Jianshe S. Yu

Copyright (C) 2010 L. Yan and B. Liu. This is an open access article distributed under the Creative Commons Attribution License, which permits unrestricted use, distribution, and reproduction in any medium, provided the original work is properly cited.

This paper studies the problem of stabilization with optimal performance for dissipative DIHS (discrete-time impulsive hybrid systems). By using Lyapunov function method, conditions are derived under which the DIHS with zero inputs is GUAS (globally uniformly asymptotically stable). These GUAS results are used to design feedback control law such that a dissipative DIHS is asymptotically stabilized and the value of a hybrid performance functional can be minimized. For the case of linear DIHS with a quadratic supply rate and a quadratic storage function, sufficient and necessary conditions of dissipativity are expressed in matrix inequalities. And the corresponding conditions of optimal quadratic hybrid performance are established. Finally, one example is given to illustrate the results.

\section{Introduction}

In many engineering problems, it is needed to consider the energy of systems. The energy of a controlled system is often linked to the concept of dissipativity [1-4]. A dissipative system here is one for which the energy dissipated inside the dynamical system is less than the energy supplied from the external source. The "energy" storage function of a dissipative system which can be viewed as generalization of energy function is often used to be a Lyapunov function, and thus the stability of a dissipative system can be investigated. It is also known that a dissipative system may be unstable. If one hopes that a dissipative but unstable system will be stable, it is necessary to use the technique of stabilization.

Feedback stabilization and dissipativity theory as well as the connected Lyapunov stability theory has been studied for systems possessing continuous motions. Byrnes et al. started to study the dissipativity and stabilization of continuous systems based on 
geometric system theory in $[5,6]$ and relevant references cited therein. Recently, notions of classical dissipativity theory have been extended for CIHS (continuous-time impulsive hybrid systems; see [7-16]), switched systems, discrete-time systems, and discontinuous systems, see [17-24]. But these reports include very few results of feedback stabilization for dissipative CIHS. The traditional methods used in the study of feedback stabilization of dissipative continuous-time systems are those based on the LaSalle invariance principle [25]. But it is difficult to use it to analyze the feedback stabilization of dissipative CIHS because solutions of impulsive hybrid systems are no longer continuous. In [14], feedback stabilization of dissipative CIHS is studied by using Lyapunov-like function, which is derived from the "energy" storage function of a dissipative CIHS. However, to the best of our knowledge, no dissipativity and feedback stabilization results have been previously reported for DIHS (discrete-time impulsive hybrid systems, see [26-28]), in which the impulses occur in discrete-time systems. Recently, in $[29,30]$ and the relevant references cited therein, the optimal control issue is also reported for CIHS and the Pontryagin-type Maximum Principle for CIHS is established. However, there are fewer results reported for stabilization with optimal performance for dissipative CIHS or DIHS.

The objective of this paper is to study the stabilization with optimal performance problem for dissipative DIHS in the spirit of $[14,20]$. By using the Lyapunov function and dwell time method, we propose some GUAS results for DIHS. Then these GUAS results are used to derive the conditions under which a dissipative DIHS is asymptotically stabilized and the hybrid performance functional is minimized.

The rest of this paper is organized as follows. In Section 2, we introduce some notations and definitions. In Section 3, we give the main results for DIHS. Then, we specialize the results to linear DIHS. Finally, in Section 4, we discuss one example to illustrate our results.

\section{Preliminaries}

Let $\mathbb{R}^{n}$ denote the $n$-dimensional Euclidean space. Let $\mathbb{R}_{+}=[0,+\infty)$ and $\mathbb{N}=\{0,1,2, \ldots\}$. A function $\phi: \mathbb{R}_{+} \rightarrow \mathbb{R}_{+}$is of class- $\mathcal{K}(\phi \in \mathcal{K})$ if it is continuous, zero at zero and strictly increasing. It is of class- $\mathcal{K}_{\infty}$ if it is of class- $\mathcal{K}$ and is unbounded. For $k_{1}, k_{2} \in \mathbb{N}$, satisfying $k_{1} \leq k_{2}$, denote $\mathcal{N}\left[k_{1}, k_{2}\right]=\left\{k: k \in \mathbb{N}, k_{1} \leq k \leq k_{2}\right\}, \mathcal{N}\left(k_{1}, k_{2}\right)=\left\{k: k \in \mathbb{N}, k_{1}<k<k_{2}\right\}$, and $\mathcal{N}\left(k_{1}, k_{2}\right]=\left\{k: k \in \mathbb{N}, k_{1}<k \leq k_{2}\right\} . X>0(X \geq 0), X \in \mathbb{R}^{n \times n}$, means that matrix $X$ is a positive definite (nonnegative definite) and symmetric matrix. Let $\|\cdot\|$ stand for the Euclidean norm in $\mathbb{R}^{n}$.

Consider the following controlled DIHS:

$$
\begin{gathered}
x(k+1)=f\left(x(k), u_{c}(k)\right), \quad k \in \supset_{i} \triangleq \mathcal{N}\left(N_{i}, N_{i+1}\right), \\
\Delta x(k)=I_{i}\left(x(k), u_{d}(k)\right), \quad k=N_{i}, \\
y_{c}(k)=h_{c}\left(x(k), u_{c}(k)\right), \quad k \in \supset_{i} \\
y_{d}(k)=h_{d}\left(x(k), u_{d}(k)\right), \quad k=N_{i}, i \in \mathbb{N},
\end{gathered}
$$

where $x(k) \in \mathbb{R}^{n}$ is the state; $y_{c}(k) \in \mathbb{R}^{l_{c}}, y_{d}(k) \in \mathbb{R}^{l_{d}}$ are the outputs; $f \in C\left[\mathbb{R}^{n} \times \mathbb{R}^{n_{c}}, \mathbb{R}^{n}\right], I_{i} \in$ $C\left[\mathbb{R}^{n} \times \mathbb{R}^{n_{d}}, \mathbb{R}^{n}\right]$ are known continuous functions with $f(0,0) \equiv 0, I_{i}(0,0) \equiv 0 ; h_{c}: \mathbb{R}^{n} \times \mathbb{R}^{n_{c}} \rightarrow$ $\mathbb{R}^{l_{c}}$ and satisfies $h_{c}(0,0)=0 ; h_{d}: \mathbb{R}^{n} \times \mathbb{R}^{n_{d}} \rightarrow \mathbb{R}^{l_{d}}$ and satisfies $h_{d}(0,0)=0 ; u_{c}: \mathbb{R}_{+} \rightarrow U_{c} \subset$ $\mathbb{R}^{n_{c}}, u_{d}: \mathbb{R}_{+} \rightarrow U_{d} \subset \mathbb{R}^{n_{d}}$ are external control inputs with $u_{c}(0)=0, u_{d}(0)=0$, here $U \triangleq$ 
$\left(U_{c}, U_{d}\right) \subset \mathbb{R}^{n_{c}} \times \mathbb{R}^{n_{d}}$ is the class of admissible hybrid control inputs; $\Delta x(k)=x(k+1)-x(k)$; and the impulsive sequence $\left\{N_{i}, i \in \mathbb{N}\right\}$ satisfie: $N_{i} \in \mathbb{N}$ and $0 \leq N_{0}<N_{1}<\cdots<N_{i}<\cdots$, with $\lim _{i \rightarrow \infty} N_{i}=\infty$ and $\Delta_{i+1} \triangleq N_{i+1}-N_{i}, i \in \mathbb{N}$. Let $x(k) \triangleq x\left(k, x_{0}, u_{c}, u_{d}\right)$ be the solution of system (2.1) with initial condition $x\left(N_{0}\right)=x_{0}$. For the impulsive sequence $\left\{N_{i}, i \in \mathbb{N}\right\}$ and any $k_{1}, k_{2} \in \mathbb{N}$ satisfying $k_{1} \leq k_{2}$, we denote $S\left[k_{1}, k_{2}\right]$ the number of impulses during $\mathcal{N}\left[k_{1}, k_{2}\right]$.

The hybrid performance functional of DIHS (2.1) is

$$
J^{k_{f}}\left(x_{0}, u_{c}, u_{d}\right)=\sum_{i=0}^{S\left[k_{0}, k_{f}\right]} \sum_{k=N_{i}+1}^{N_{i+1}-1} L_{c}\left(x(k), u_{c}(k)\right)+\sum_{i=0}^{S\left[k_{0}, k_{f}\right]} L_{d}\left(x\left(N_{i}\right), u_{d}\left(N_{i}\right)\right),
$$

where $k_{0}, k_{f} \in \mathbb{N}$ with $k_{0}=N_{0}, k_{f}<\infty$, or $k_{f}=\infty$, and $L_{c}, L_{d}$ are given and known functions.

Remark 2.1. (i) If there exists a positive integer $\widehat{k}$ such that $\Delta_{\widehat{k}+1}=+\infty$, then (2.1) becomes a normal discrete-time system with initial point $\left(N_{0}=N_{\hat{k}}, x_{0}\right)$. In this paper, we study the DIHS under the following assumption:

$$
2 \leq \Delta_{\text {inf }} \triangleq \inf _{i \in \mathbb{N}}\left\{\Delta_{i}\right\} \leq \Delta_{\text {sup }} \triangleq \sup _{i \in \mathbb{N}}\left\{\Delta_{i}\right\}<\infty
$$

(ii) By (2.3), we get the fact that for any $k \in \mathcal{N}\left(N_{i}, N_{i+1}\right], i \in \mathbb{N}, k \rightarrow \infty$ if and only if $i \rightarrow \infty$.

Definition 2.2. A function $\left(\gamma_{c}\left(u_{c}, y_{c}\right), \gamma_{d}\left(u_{d}, y_{d}\right)\right)$, where $\gamma_{c}: \mathbb{R}^{m_{c}} \times \mathbb{R}^{l_{c}} \rightarrow \mathbb{R}, \gamma_{d}: \mathbb{R}^{m_{d}} \times \mathbb{R}^{l_{d}} \rightarrow \mathbb{R}$ with $\gamma_{c}(0,0)=0$ and $\gamma_{d}(0,0)=0$, is called a supply rate of (2.1) if $\gamma_{c}\left(u_{c}, y_{c}\right)$ and $\gamma_{d}\left(u_{d}, y_{d}\right)$ are locally summable: for all input-output pairs $(u, y)$ and any $k_{1}, k_{2} \in \mathbb{N}$ with $k_{1} \leq k_{2}, \gamma_{c}, \gamma_{d}$ satisfy $\sum_{k_{1} \leq k<k_{2}}\left|\gamma_{c}\left(u_{c}(k), y_{c}(k)\right)\right|<\infty, \sum_{k_{1} \leq k<k_{2}}\left|\gamma_{d}\left(u_{d}(k), y_{d}(k)\right)\right|<\infty$.

Definition 2.3. DIHS (2.1) is said to be dissipative under supply rate $\left(\gamma_{c}, \gamma_{d}\right)$ if there exists a nonnegative continuous function $V: \mathbb{R}^{n} \rightarrow \mathbb{R}_{+}$with $V(0)=0$, called storage function, such that for all $\left(u_{c}, u_{d}\right) \in U$ the following dissipation inequality holds for any $\widehat{k}, k \in \mathbb{N}$ with $N_{0} \leq \widehat{k} \leq k$,

$$
\begin{aligned}
V(x(k)) \leq & V(x(\widehat{k}))+\sum_{i=S[\widehat{k}, k]}^{k-1} \gamma_{c}\left(u_{c}(j), y_{c}(j)\right)+\sum_{i=0}^{S[\hat{k}, k]} \sum_{j=N_{i}+1}^{N_{i+1}-1} \gamma_{c}\left(u_{c}(j), y_{c}(j)\right) \\
& +\sum_{i=0}^{S[\hat{k}, k]} \gamma_{d}\left(u_{d}\left(N_{i}\right), y_{d}\left(N_{i}\right)\right) .
\end{aligned}
$$

Lemma 2.4. DIHS (2.1) is dissipative under the supply rate $\left(\gamma_{c}, \gamma_{d}\right)$ if and only if there exists a nonnegative continuous function $V$ with $V(0)=0$ such that

$$
\begin{gathered}
\Delta V(x(k)) \leq \gamma_{c}\left(u_{c}(k), y_{c}(k)\right), \quad k \in \supset_{i}, \\
\Delta V(x(k)) \leq \gamma_{d}\left(u_{d}(k), y_{d}(k)\right), \quad k=N_{i}, i \in \mathbb{N},
\end{gathered}
$$

where $\Delta V(x(k))=V(x(k+1))-V(x(k)), \quad k \in \mathbb{N}$. 
Proof. By using Definition 2.3, it is easy to get that (2.4) is equivalent to (2.5). The details are omitted here.

\subsection{Stabilization with Optimal Performance Problem}

For the dissipative DIHS (2.1) with hybrid performance functional (2.2), the stabilization with optimal performance problem is to design the state feedback control law $\left(u_{c}, u_{d}\right)=$ $\left(\phi_{c}(x), \phi_{d}(x)\right)$, where $\phi_{c}: \mathbb{R}^{n} \rightarrow \mathbb{R}^{n_{c}}, \phi_{d}: \mathbb{R}^{n} \rightarrow \mathbb{R}^{n_{d}}$ with $\phi_{c}(0)=0, \phi_{d}(0)=0$, such that the closed-loop system

$$
\begin{gathered}
x(k+1)=f\left(x(k), \phi_{c}(x(k))\right), \quad k \in \mathcal{O}_{i}=\mathcal{N}\left(N_{i}, N_{i+1}\right), \\
\Delta x=I_{i}\left(x(k), \phi_{d}(x(k))\right), \quad k=N_{i}, i \in \mathbb{N}, \\
x\left(N_{0}\right)=x_{0}
\end{gathered}
$$

is GUAS. Moreover, $\left(u_{c}(k), u_{d}(k)\right)=\left(\phi_{c}(x(k)), \phi_{d}(x(k))\right)$ can minimize $J^{k_{f}}\left(x_{0}, u_{c}, u_{d}\right)$.

\section{Main Results}

In this section, by using the Lyapunov function method, some GUAS criteria are established for DIHS. Then, these stability criteria are used to study the optimal stabilization issue for a dissipative DIHS with hybrid performance functional.

Theorem 3.1. Let $\left(u_{c}, u_{d}\right) \equiv 0$. Suppose (2.3) holds and furthermore assume that there exists a function $V \in C\left[\mathbb{R}^{n}, \mathbb{R}_{+}\right]$such that

(i) there exist $\mathcal{K}_{\infty}$-functions $c_{1}, c_{2}$ such that for any $x \in \mathbb{R}^{n}$,

$$
c_{1}(\|x\|) \leq V(x) \leq c_{2}(\|x\|)
$$

(ii) there exists a $\phi_{1} \in \mathcal{K}$ satisfying $\phi_{1}<\mathbf{1}$ and

$$
V(x(k+1))-V(x(k)) \leq-\phi_{1}(V(x(k))), \quad k \in \supset_{i}, i \in \mathbb{N},
$$

where $\mathbf{1}$ is the identity function: $\mathbf{1}(s)=s$ for any $s \in \mathbb{R}_{+}$;

(iii) for $k=N_{i}, i \in \mathbb{N}$, there exists $\mathcal{K}$-function $\phi_{2}$ such that

$$
V\left(x\left(N_{i}+1\right)\right) \leq \phi_{2}\left(V\left(x\left(N_{i}\right)\right)\right) ;
$$

(iv) there exists a sufficient large $\Delta_{\text {inf }}>1$, such that

$$
\left(\mathbf{1}-\phi_{1}\right)^{\Delta_{\mathrm{inf}}-1} \circ \phi_{2}<\mathbf{1} .
$$

Then, DIHS (2.1) with $\left(u_{c}, u_{d}\right) \equiv 0$ is GUAS. 
Proof. Denote $\phi \triangleq\left(\mathbf{1}-\phi_{1}\right)^{\Delta_{\mathrm{inf}}-1} \circ \phi_{2}$. By condition (ii), we get that, for any $k \in \mathcal{O}_{i}=\mathcal{N}\left(N_{i}, N_{i+1}\right)$,

$$
V(x(k+1)) \leq\left(\mathbf{1}-\phi_{1}\right)^{k-N_{i}-1}\left(V\left(x\left(N_{i}+1\right)\right)\right) .
$$

It follows from (3.5) and condition (iii) that

$$
\begin{aligned}
V\left(x\left(N_{i+1}\right)\right) & \leq\left(\mathbf{1}-\phi_{1}\right)^{N_{i+1}-N_{i}-1}\left(V\left(x\left(N_{i}+1\right)\right)\right) \\
& \leq\left(\mathbf{1}-\phi_{1}\right)^{N_{i+1}-N_{i}-1}\left(\phi_{2}\left(V\left(x\left(N_{i}\right)\right)\right) \leq \phi\left(V\left(x\left(N_{i}\right)\right)\right), \quad i \in \mathbb{N} .\right.
\end{aligned}
$$

For any $k \in \mathbb{N}$, there exists an $i \in \mathbb{N}$ such that $k \in \mathcal{N}\left(N_{i}, N_{i+1}\right]$. By (3.6) and conditions (i)-(iii), we have

$$
\|x(k)\| \leq c_{1}^{-1}\left(\phi_{2}\left(V\left(x\left(N_{i}\right)\right)\right) \leq \mathrm{c}_{1}^{-1}\left(\phi_{2}\left(c_{2}\left(\left\|x_{0}\right\|\right)\right)\right) .\right.
$$

Hence, for any $\epsilon>0$, let $0<\delta<c_{2}^{-1}\left(\phi_{2}^{-1}\left(c_{1}(\epsilon)\right)\right)$; then, when $\left\|x_{0}\right\| \leq \delta$, we get from (3.7) that $\|x(k)\|<\epsilon$ for any $k \in \mathbb{N}$. Thus, the system (2.1) is GUS (globally uniformly stable).

Denote $a_{i}=V\left(x\left(N_{i}\right)\right), i \in \mathbb{N}$. It follows from (3.6) that

$$
a_{i+1} \leq \phi\left(a_{i}\right), \quad i \in \mathbb{N}
$$

Since by condition (iv), $\phi(s)<s$ for any $s>0$, thus we get that the sequence $\left\{a_{i}, i \in \mathbb{N}\right\}$ is monotone decreasing and $\lim _{i \rightarrow \infty} a_{i}=a$ exists. If $a>0$, then, $a=\lim _{i \rightarrow \infty} a_{i+1}=\lim _{i \rightarrow \infty} \phi\left(a_{i}\right)=$ $\phi(a)<a$. This contradiction implies $a=0$, that is, $\lim _{i \rightarrow \infty} a_{i}=0$.

For any $k \in \mathcal{N}\left(N_{i}, N_{i+1}\right]$, by conditions (i)-(iii), we have $\|x(k)\| \leq c_{1}^{-1}(V(x(k))) \leq$ $c_{1}^{-1}\left(\phi_{2}\left(a_{i}\right)\right)$. It follows from Remark 2.1(ii) that $\lim _{k \rightarrow \infty}\|x(k)\|=0$. Hence, DIHS (2.1) with $\left(u_{c}, u_{d}\right) \equiv 0$ is uniformly attractive and hence it is GUAS. The proof is complete.

Theorem 3.2. Let $\left(u_{c}, u_{d}\right) \equiv 0$ and suppose (2.3) holds and furthermore assume that there exists a $V(x)$ satisfying conditions (i) and (iii) of Theorem 3.1 and

(ii*) there exists a $\phi_{1} \in \mathcal{K}$ such that

$$
V(x(k+1))-V(x(k)) \leq \phi_{1}(V(x(k))), \quad k \in \supset_{i}, i \in \mathbb{N}
$$

$\left(\mathrm{iv}^{*}\right)$ there exists a sufficient large $\Delta_{\text {sup }}>1$, such that

$$
\left(\mathbf{1}+\phi_{1}\right)^{\Delta_{\text {sup }}-1} \circ \phi_{2}<\mathbf{1}
$$

Then, DIHS (2.1) with $\left(u_{c}, u_{d}\right) \equiv 0$ is GUAS.

Proof. By similar proof of Theorem 3.1 with $\phi \triangleq\left(1+\phi_{1}\right)^{\Delta_{\text {sup }}-1} \circ \phi_{2}$, we obtain that the result holds. The detailed is omitted here.

Corollary 3.3. Let $\left(u_{c}, u_{d}\right) \equiv 0$ and suppose (2.3) holds and assume that there exists a function $V \in C\left[\mathbb{R}^{n}, \mathbb{R}_{+}\right]$satisfying (3.1) and 
(i) there exists a constant $a \in R$ satisfying $a>-1$ and

$$
V(x(k+1))-V(x(k)) \leq a V(x(k)), \quad k \in \supset_{i}, i \in \mathbb{N} ;
$$

(ii) for $k=N_{i}, i \in \mathbb{N}$, there exists a constant $b>0$ such that

$$
V\left(x\left(N_{i}+1\right)\right) \leq b V\left(x\left(N_{i}\right)\right)
$$

(iii) one of the following cases holds.

Case 1. There exists a sufficient large $\Delta_{\text {inf }}>1$, such that

$$
(1+a)^{\Delta_{\text {inf }}-1} b<1 \quad \text { if }-1<a<0, b>0 .
$$

Case 2. There exists a sufficient large $\Delta_{\text {sup }}>1$, such that

$$
(1+a)^{\Delta_{\text {sup }}-1} b<1 \quad \text { if } a \geq 0,0<b<1 .
$$

Then, DIHS (2.1) with $\left(u_{c}, u_{d}\right) \equiv 0$ is GUAS.

Proof. The result is the direct consequence of Theorems 3.1 and 3.2, where in Case 1, let $\phi_{1}(s)=$ -as, $\phi_{2}(s)=b s$, while in Case 2 , let $\phi_{1}(s)=$ as and $\phi_{2}(s)=b s$ for any $s \in \mathbb{R}_{+}$.

Remark 3.4. Theorems 3.1 and 3.2 and Corollary 3.3 give two kinds of GUAS properties of DIHS by using the method of Lyapunov function and maximal and minimal dwell times $\Delta_{\text {sup }}, \Delta_{\text {inf }}$. For more detailed stability results of DIHS, please refer to the literature [26-28] and relevant references cited therein.

Theorem 3.5. Suppose (2.3) holds and assume that under the given supply rate $\left(\gamma_{c}, \gamma_{d}\right)$, DIHS (2.1) is dissipative with a storage function $V(x)$ satisfying (3.1), and that there exist functions $\phi_{c}: \mathbb{R}^{n} \rightarrow$ $\mathbb{R}^{n_{c}}$ and $\phi_{d}: \mathbb{R}^{n} \rightarrow \mathbb{R}^{n_{d}}$ with $\phi_{c}(0)=0$ and $\phi_{d}(0)=0$, such that

(i) there exist $\phi_{1}, \phi_{2} \in \mathcal{K}$ with $\phi_{1}<\mathbf{1}$ satisfying (3.4) and

$$
\begin{aligned}
& \gamma_{c}\left(\phi_{c}(x(k)), y_{c}(k)\right) \leq-\phi_{1}(V(x(k))), \quad k \in \supset_{i}, \\
& \gamma_{d}\left(\phi_{d}\left(x\left(N_{i}\right), y_{d}\left(N_{i}\right)\right)\right) \leq\left(\phi_{2}-\mathbf{1}\right)\left(V\left(x\left(N_{i}\right)\right)\right) ;
\end{aligned}
$$

(ii) the following equations and inequalities are satisfied:

$$
\begin{gathered}
\left.H_{c}\left(x, u_{c}\right)\right|_{u_{c}=\phi_{c}(x)}=0, \quad k \in \supset_{i}, \quad i \in \mathbb{N}, \\
\left.H_{d i}\left(x, u_{d}\right)\right|_{u_{d}=\phi_{d}(x)}=0, \quad k=N_{i}, \quad i \in \mathbb{N}, \\
H_{c}\left(x, u_{c}\right) \geq 0, \quad \forall u_{c} \in U_{c}, \\
H_{d i}\left(x, u_{d}\right) \geq 0, \quad \forall u_{d} \in U_{d}, \quad i \in \mathbb{N},
\end{gathered}
$$

where $H_{c}\left(x, u_{c}\right) \triangleq L_{c}\left(x, u_{c}\right)-V(x)+V\left(f\left(x, u_{c}\right)\right)$ and $H_{d i}\left(x, u_{d}\right) \triangleq L_{d}\left(x, u_{d}\right)+V(x+$ $\left.I_{i}\left(x, u_{d}\right)\right)-V(x)$. 
Then, under $\left(u_{c}(k), u_{d}(k)\right)=\left(\phi_{c}(x(k)), \phi_{d}(x(k))\right), k \in \mathbb{N}$, the closed-loop system (2.6) is GUAS, and

$$
J^{k_{f}}\left(x_{0}, \phi_{c}(x), \phi_{d}(x)\right) \leq V\left(x_{0}\right)
$$

Specially, $J^{\infty}\left(x_{0}, \phi_{c}(x), \phi_{d}(x)\right)=V\left(x_{0}\right)$.

Proof. Since system (2.1) is dissipative under the supply rate $\left(\gamma_{c}, \gamma_{d}\right)$, then, for $\left(u_{c}, u_{d}\right)=$ $\left(\phi_{c}(x), \phi_{d}(x)\right)$, we get

$$
\begin{gathered}
\left.\Delta V(x(K))\right|_{u_{c}=\phi_{c}(x)} \leq \gamma_{c}\left(\phi_{c}(x(k)), y_{c}(k)\right), \quad k \in \supset_{i}, \\
\left.\Delta V\left(x\left(N_{i}\right)\right)\right|_{u_{d}=\phi_{d}(x)} \leq \gamma_{d}\left(\phi_{d}\left(x\left(N_{i}\right)\right), y_{d}\left(N_{i}\right)\right), \quad i \in \mathbb{N} .
\end{gathered}
$$

From condition (i) and (3.19), we derive that

$$
\begin{gathered}
\left.\Delta V(x(K))\right|_{u_{c}=\phi_{c}(x)} \leq-\phi_{1}(V(x(k))), \quad k \in \supset_{i}, \\
\left.V\left(x\left(N_{i}+1\right)\right)\right|_{u_{d}=\phi_{d}(x)} \leq \phi_{2}\left(V\left(x\left(N_{i}\right)\right)\right), \quad i \in \mathbb{N} .
\end{gathered}
$$

Thus, from (3.20) and Theorem 3.1, we obtain that the closed-loop system (2.6) is GUAS.

By condition (ii), for $k \in \mathcal{N}\left(N_{i}, N_{i+1}\right], i \in \mathbb{N}$, we get

$$
\begin{array}{cl}
L_{c}\left(x(k), \phi_{c}(x(k))\right)=-\Delta V(x(k)), & k \in \supset_{i}, \\
L_{d}\left(x(k), \phi_{d}(x(k))\right)=-\Delta V(x(k)), & k=N_{i} .
\end{array}
$$

Denote $x_{f}=x\left(N_{S\left[k_{0}, k_{f}\right]}\right)$. From (3.21), we have

$$
\begin{aligned}
J^{k_{f}}\left(x_{0}, \phi_{c}, \phi_{d}\right)= & \sum_{i=0}^{S\left[k_{0}, k_{f}\right]} \sum_{k=N_{i}+1}^{N_{i+1}-1} L_{c}\left(x(k), u_{c}(k)\right)+\sum_{i=0}^{S\left[k_{0}, k_{f}\right]} L_{d}\left(x\left(N_{i}\right), u_{d}\left(N_{i}\right)\right) \\
= & \sum_{i=0}^{S\left[k_{0}, k_{f}\right]} \sum_{k=N_{i}+1}^{N_{i+1}-1}-\Delta V(x(k))-\sum_{i=0}^{S\left[k_{0}, k_{f}\right]} \Delta V\left(x\left(N_{i}\right)\right) \\
= & -\sum_{i=0}^{S\left[k_{0}, k_{f}\right]} \sum_{k=N_{i}+1}^{N_{i+1}-1}\left(V\left(x\left(N_{i+1}\right)\right)-V\left(x\left(N_{i}+1\right)\right)\right) \\
& -\sum_{i=0}^{S\left[k_{0}, k_{f}\right]}\left(V\left(x\left(N_{i}+1\right)\right)-V\left(x\left(N_{i}\right)\right)\right) \\
= & \sum_{i=0}^{S\left[k_{0}, k_{f}\right]}\left(V\left(x\left(N_{i}\right)\right)-V\left(x\left(N_{i+1}\right)\right)\right) \\
= & V\left(x_{0}\right)-V\left(x_{f}\right) \leq V\left(x_{0}\right) .
\end{aligned}
$$


Thus, from (3.22) condition (i), and the fact that the closed-loop system is GUAS, we obtain that

$$
J^{k_{f}}\left(x_{0}, \phi_{c}, \phi_{d}\right) \leq V\left(x_{0}\right), \quad J^{\infty}\left(x_{0}, \phi_{c}, \phi_{d}\right)=V\left(x_{0}\right)
$$

Now, we prove that $\left(u_{c}, u_{d}\right)=\left(\phi_{c}(x), \phi_{d}(x)\right)$ minimizes $J^{k_{f}}\left(x_{0}, u_{c}, u_{d}\right)$. From condition (ii), we have

$$
\begin{gathered}
L_{c}\left(x(k), u_{c}(k)\right)=-\Delta V(x(k))+H_{c}\left(x(k), u_{c}(k)\right), \\
L_{d}\left(x(k), u_{d}(k)\right)=-\Delta V(x(k))+H_{d i}\left(x(k), u_{d}(k)\right) .
\end{gathered}
$$

Thus, using (3.24), (3.22), and $H_{c} \geq 0, H_{d i} \geq 0$, we have

$$
\begin{aligned}
J^{k_{f}}\left(x_{0}, u_{c}, \mathrm{u}_{d}\right)= & \sum_{i=0}^{S\left[k_{0}, k_{f}\right]} \sum_{k=N_{i}+1}^{N_{i+1}-1}\left(-\Delta V(x(k))+H_{c}\left(x(k), u_{c}(k)\right)\right) \\
& +\sum_{i=0}^{S\left[k_{0}, k_{f}\right]}\left(-\Delta V\left(x\left(N_{i}\right)\right)+H_{d i}\left(x\left(N_{i}\right), u_{d}\left(N_{i}\right)\right)\right) \\
\geq & V\left(x_{0}\right)-V\left(x_{f}\right)=J^{k_{f}}\left(x_{0}, \phi_{c}(x), \phi_{d}(x)\right)
\end{aligned}
$$

Hence, (3.18) holds and all the results hold.

Theorem 3.6. Suppose (2.3) holds and assume that under the supply rate $\left(\gamma_{c}, \gamma_{d}\right)$, system (2.1) is dissipative with a storage function $V(x)$ satisfying (3.1), and that there exist functions $\phi_{c}$, $\phi_{d}$ with $\phi_{c}(0)=0, \phi_{d}(0)=0$, such that (ii) of Theorem 3.5 holds while (i) of Theorem 3.5 is replaced by the following:

$\left(\mathrm{i}^{*}\right)$ there exist $\phi_{1}, \phi_{2} \in \mathcal{K}$ with $\phi_{2}<\mathbf{1}$ satisfying (3.10) and

$$
\begin{gathered}
\gamma_{c}\left(\phi_{c}(x(k)), y_{c}(k)\right) \leq \phi_{1}(V(x(k))), \quad k \in \supset_{i}, \\
\gamma_{d}\left(\phi_{d}\left(x\left(N_{i}\right), y_{d}\left(N_{i}\right)\right)\right) \leq\left(\phi_{2}-\mathbf{1}\right)\left(V\left(x\left(N_{i}\right)\right)\right) .
\end{gathered}
$$

Then, all results of Theorem 3.5 still hold.

Proof. By similar proof of Theorem 3.5 and using the result of Theorem 3.2, we obtain that all results are true.

Remark 3.7. (i) For a dissipative DIHS (2.1) with supply rate $\left(\gamma_{c}, \gamma_{d}\right)$ and "energy" storage function $V$, if $\gamma_{c}$ or $\gamma_{d}$ is negative during some time interval or at some time instance, then it implies that the "energy" of system will be decreasing during this period or at this instance. These two kinds of dissipativity properties all help to achieve the stability for whole DIHS. In Theorem 3.5, the negative supply rate $\gamma_{c}$ leads to the decreasing of "energy" of system during two consecutive impulses (see (3.15)) and thus it permits to some extend the increasing of "energy" at impulsive instances (see (3.16)) while the stability property of whole system will be kept. On the other hand, in Theorem 3.6, the negative supply rate $\gamma_{d}$ leads to the decreasing 
of "energy" of system at impulse instances (see (3.27)) and thus it permits to some extend of increasing of "energy" during two consecutive impulses (see (3.26)) while the stability property of whole system can still be guaranteed.

In the literature, if the stability property is derived from the dissipativity of system, it often needs the condition of negative supply rate. But one can see from Theorems 3.5 and 3.6 that this condition is relaxed for DIHS.

(ii) By (3.17), if $H_{c}\left(x, u_{c}\right)$ and $H_{d i}\left(x, u_{d}\right)$ are continuously differential in $u_{c}$ and $u_{d}$, respectively, then, for $i \in \mathbb{N}$,

$$
\left.\frac{\partial H_{c}\left(x, u_{c}\right)}{\partial u_{c}}\right|_{u_{c}=\phi_{c}(x)}=0,\left.\quad \frac{H_{d i}\left(x, u_{d}\right)}{\partial u_{d}}\right|_{u_{d}=\phi_{d}(x)}=0
$$

which can be used to derive the hybrid state feedback control law $\left(u_{c}, u_{d}\right)=\left(\phi_{c}(x), \phi_{d}(x)\right)$.

At the end of section, we specialize the results obtained to the case of linear DIHS with a quadratic supply rate.

Consider the following linear DIHS:

$$
\begin{gathered}
x(k+1)=A_{c} x(k)+B_{c} u_{c}(k), \quad k \in O_{i}, \\
\Delta x(k)=\left(A_{d}-I_{n}\right) x(k)+B_{d} u_{d}(k), \quad k=N_{\mathrm{i}}, \\
y_{c}(k)=C_{c} x(k)+D_{c} u_{c}(k), \quad k \in O_{i}, \\
y_{d}(k)=C_{d} x(k)+D_{d} u_{d}(k), \quad k=N_{i}, \quad i \in \mathbb{N},
\end{gathered}
$$

with the hybrid quadratic performance functional:

$$
\begin{aligned}
J^{k_{f}}\left(x_{0}, u_{c}, u_{d}\right)= & \sum_{i=0}^{S\left[k_{0}, k_{f}\right]} \sum_{k=N_{i}+1}^{N_{i+1}-1}\left[x^{T}(k) P_{c} x(k)+u_{c}^{T}(k) T_{c} u_{c}(k)\right] \\
& +\sum_{i=0}^{S\left[k_{0}, k_{f}\right]}\left[x\left(N_{i}\right)^{T} P_{d} x\left(N_{i}\right)+u_{d}^{T}\left(N_{i}\right) T_{d} u_{d}\left(N_{i}\right)\right]
\end{aligned}
$$

where $A_{c}, B_{c}, C_{c}, D_{c}, A_{d}, B_{d}, C_{d}, D_{d}, P_{c}, P_{d}, T_{c}$, and $T_{d}$ are matrices with appropriate dimensions and $T_{c}>0$ and $T_{d}>0$.

The quadratic supply rate $\left(\gamma_{c}, \gamma_{d}\right)$ is given by

$$
\begin{gathered}
\gamma_{c}\left(u_{c}, y_{c}\right)=y_{c}^{T} R_{c} y_{c}+2 y_{c}{ }^{T} S_{c} u_{c}+u_{c}^{T} Q_{c} u_{c}, \\
\gamma_{d}\left(u_{d}, y_{d}\right)=y_{d}^{T} R_{d} y_{d}+2 y_{d}^{T} S_{d} u_{d}+u_{d}^{T} Q_{d} u_{d},
\end{gathered}
$$

where $R_{c}, S_{c}, Q_{c}, R_{d}, S_{d}$, and $Q_{d}$ are matrices with appropriate dimensions and $R_{c}, R_{d}$, $Q_{c}$, and $Q_{d}$ are symmetric matrices.

Denote $X_{c} \triangleq Q_{c}+S_{c} D_{c}+D_{c}^{T} S_{c}+D_{c}^{T} R_{c} D_{c}$ and $X_{d} \triangleq Q_{d}+S_{d} D_{d}+D_{d}^{T} S_{d}+D_{d}^{T} R_{d} D_{d}$. 
Theorem 3.8. Assume that $X_{c} \geq 0, X_{d} \geq 0$, and for $\left(\gamma_{c}, \gamma_{d}\right)$, the linear DIHS (3.29) is dissipative with storage function $V(x)=x^{T} X x$, where $X>0$, if and only if the following LMIs are satisfied:

$$
\Psi_{z} \triangleq\left(\begin{array}{cccc}
-X-C_{z}^{T} R_{z} C_{z} & -C_{z}^{T}\left(R_{z} D_{z}+S_{z}\right) & \frac{1}{2} A_{z}^{T} X & 0 \\
-\left(S_{z}+D_{z}^{T} R_{z}\right) C_{z} & -X_{z} & 2 B_{z}^{T} X & \frac{1}{2} B_{z}^{T} X \\
\frac{1}{2} X A_{z} & 2 X B_{z} & -X & 0 \\
0 & \frac{1}{2} X B_{z} & 0 & -X
\end{array}\right) \leq 0, \quad z=c, d
$$

Moreover, if $K_{c}=-\left(T_{c}+B_{c}^{T} X B_{c}\right)^{-1} B_{c}^{T} X A_{c}$ and $K_{d}=-\left(T_{d}+B_{d}^{T} X B_{d}\right)^{-1} B_{d}^{T} X A_{d}$ satisfy

$$
\begin{gathered}
K_{c}{ }^{T} X_{c} K_{c}+K_{c}{ }^{T}\left(S_{c}+D_{c}^{T} R_{c}\right) C_{c}+C_{c}^{T}\left(S_{c}+R_{c} D_{c}\right) K_{c}+C_{c}{ }^{T} R_{c} C_{c}-a \cdot X \leq 0 \\
K_{d}{ }^{T} X_{d} K_{d}+K_{d}{ }^{T}\left(S_{d}+D_{d}^{T} R_{d}\right) C_{d}+C_{d}^{T}\left(S_{d}+R_{d} D_{d}\right) K_{d}+C_{d}^{T} R_{d} C_{d}-(b-1) \cdot X \leq 0 \\
A_{c}^{T} X A_{c}+P_{c}-X-A_{c}^{T} X B_{c} K_{c}=0 \\
A_{d}^{T} X A_{d}+P_{c}-X-A_{d}^{T} X B_{d} K_{d}=0
\end{gathered}
$$

where $a, b$ satisfy $a>-1, b \geq 0$, and the condition (iii) of Corollary 3.3, then, the state feedback control law

$$
\left(u_{c}, u_{d}\right)=\left(K_{c} x, K_{d} x\right)
$$

can stabilize system (3.29), and minimizes $J^{k_{f}}\left(x_{0}, u_{c}, u_{d}\right)$, that is,

$$
J^{t_{f}}\left(x_{0}, K_{c} x, K_{d} x\right) \leq x_{0}^{T} X x_{0}=J^{\infty}\left(x_{0}, K_{c} x, K_{d} x\right) .
$$

Proof. By (3.29), it is not difficult to get that

$$
\begin{gathered}
\Delta V(x(k))-\gamma_{c}\left(u_{c}(k), y_{c}(k)\right)=\left(x^{T}, u_{c}^{T}\right) \Lambda_{c}\left(x^{T}, u_{c}^{T}\right)^{T}, \\
\Delta V(x(k))-\gamma_{d}\left(u_{d}(k), y_{d}(k)\right)=\left(x^{T}, u_{d}^{T}\right) \Lambda_{d}\left(x^{T}, u_{d}^{T}\right)^{T},
\end{gathered}
$$

where for $z=c, d, \Lambda_{z}=\left(\begin{array}{c}\Lambda_{z 1} \\ \Lambda_{z 2}^{T} \Lambda_{z 3}\end{array}\right)$, and $\Lambda_{z 1}=A_{z}{ }^{T} X A_{z}-X-C_{z}{ }^{T} R_{z} C_{z}, \Lambda_{z 2}=A_{z}{ }^{T} X B_{z}-$ $C_{z}^{T}\left(R_{z} D_{z}+S_{z}\right), \Lambda_{z 3}=B_{z}{ }^{T} X B_{z}-X_{z}$.

Thus, by Lemma 2.4, we get that system (3.29) is dissipative if and only if $\Lambda_{c} \leq$ 0 and $\Lambda_{d} \leq 0$. By Schur Complement Theorem [31], for $z=c, d$, it is not hard to get that $\Lambda_{z} \leq 0$ if and only if LMI $\Psi_{z} \leq 0$ holds. Hence, we obtain that system (3.29) is dissipative if and only if LMIs (3.32) $\Psi_{z} \leq 0, z=c, d$, hold. 
Let $u_{c}(t)=K_{c} x(t)$ and $(t)=K_{d} x(t)$, where $K_{c}=-\left(T_{c}+B_{c}^{T} X B_{c}\right)^{-1} B_{c}^{T} X A_{c}$ and $K_{d}=$ $-\left(T_{d}+B_{d}^{T} X B_{d}\right)^{-1} B_{d}^{T} X A_{d}$; then, it follows from (3.33) that for $i \in \mathbb{N}$,

$$
\begin{gathered}
\left.\Delta V(x(k))\right|_{u_{c}=K_{c} x} \leq\left.\gamma_{c}\left(u_{c}, y_{c}(k)\right)\right|_{u_{c}=K_{c} x} \leq a \cdot V(x(k)), \quad k \in \supset_{i}, \\
\Delta V(x(k)) \leq\left.\gamma_{d}\left(u_{d}, y_{d}\right)\right|_{u_{d}=K_{d} x} \leq(b-1) \cdot V(x(k)), \quad k=N_{i} .
\end{gathered}
$$

Thus, by Corollary 3.3, the closed-loop system given by (3.29) and (3.35) is GUAS.

Now, we show that (3.35) also minimizes $J^{k_{f}}\left(x_{0}, u_{c}, u_{d}\right)$.

Denote: $L_{c}\left(x, u_{c}\right) \triangleq x^{T}(k) P_{c}(k) x(k)+u_{c}^{T}(k) T_{c}(k) u_{c}(k)$ and $L_{d}\left(x, u_{d}\right) \triangleq$ $x^{T}(k) P_{d}(k) x(k)+u_{\mathrm{d}}^{T}(k) T_{d}(k) u_{d}(k)$. Then, by (3.34), we obtain

$$
\begin{aligned}
H_{c}\left(x, u_{c}\right) \triangleq & L_{c}\left(x, u_{c}\right)+V\left(A_{c} x+B_{c} u_{c}\right)-V(x) \\
= & \left(\begin{array}{c}
x \\
u_{c}
\end{array}\right)^{T}\left(\begin{array}{cc}
A_{c}^{T} X A_{c}+P_{c}-X & A_{c}^{T} X B_{c} \\
B_{c}^{T} X A_{c} & T_{c}+B_{c}^{T} X B_{c}
\end{array}\right)\left(\begin{array}{c}
x \\
u_{c}
\end{array}\right) \\
= & {\left[\left(T_{c}+B_{c}^{T} X B_{c}\right)^{-1} B_{c}^{T} X A_{c} x+u_{c}\right]^{T}\left(T_{c}+B_{c}^{T} X B_{c}\right) } \\
& \cdot\left[\left(T_{c}+B_{c}^{T} X B_{c}\right)^{-1} B_{c}^{T} X A_{c} x+u_{c}\right], \\
H_{d}\left(x, u_{d}\right) \triangleq & L_{d}\left(x, u_{d}\right)+V\left(A_{d} x+B_{d} u_{d}\right)-V(x) \\
= & {\left[\left(T_{d}+B_{d}^{T} X B_{d}\right)^{-1} B_{d}^{T} X A_{d} x+u_{d}\right]^{T}\left(T_{d}+B_{d}^{T} X B_{d}\right) } \\
& \cdot\left[\left(T_{d}+B_{d}^{T} X B_{d}\right)^{-1} B_{d}^{T} X A_{d} x+u_{d}\right] .
\end{aligned}
$$

Hence, by $T_{c}>0, T_{d}>0$, we get

$$
H_{c}\left(x, u_{c}\right) \geq 0, \quad u_{c} \in U_{c} ; \quad H_{d}\left(x, u_{d}\right) \geq 0, \quad u_{d} \in U_{d}
$$

Clearly, if $u_{c}=K_{c} x, u_{d}=K_{d} x$, then, by (3.39), we have

$$
\left.H_{c}\left(x, u_{c}\right)\right|_{u_{c}=K_{c} x}=0,\left.\quad H_{d}\left(x, u_{d}\right)\right|_{u_{d}=K_{d} x}=0 .
$$

Then, by Theorem 3.5, the result of this theorem follows readily. The proof is complete.

Corollary 3.9. Assume that $X_{c}>0, X_{d}>0$, and there exists a matrix $X>0$ satisfying LMI (3.32), (3.34), and the following matrix inequalities:

$$
\Phi_{z} \triangleq\left(\begin{array}{cc}
\Phi_{z 1}(\mu) & \Phi_{z 2} \\
\Phi_{z 2}^{T} & \Phi_{z 3}
\end{array}\right) \leq 0, \quad z=c, d,
$$


where $\Phi_{z 1}=C_{z}^{T} R_{z} C_{z}-\mu X, \Phi_{z 2}=\left(K_{z}+X_{z}^{-1}\left(S_{z}+D_{z}^{T} R_{z}\right) C_{z}\right)^{T}, \Phi_{z 3}=-X_{z}^{-1}$, and $\mu=a$ if $z=c$, $\mu=b-1$ if $z=d$; and $K_{z}=-\left(T_{z}+B_{z}^{T} X B_{z}\right)^{-1} B_{z}^{T} X A_{z}$ for $z=c$, d; and where constants $a, b$ satisfy the condition (iii) of Corollary 3.3.

Then, all the results of Theorem 3.8 still hold.

Proof. By Schur Complement Theorem [31] and Theorem 3.8, the result of this corollary follows.

\section{Examples}

In this section, one example is solved to illustrate the obtained results.

Example 4.1. Consider DIHS in form of (3.29) where

$$
\begin{gathered}
A_{c}=\left(\begin{array}{ccc}
-0.5 & 0 & 0 \\
0 & -1 & -0.5 \\
-1 & 0 & -0.5
\end{array}\right), \quad B_{c}=\left(\begin{array}{l}
0.1 \\
0.1 \\
0.2
\end{array}\right), \quad C_{c}=\left(\begin{array}{lll}
1 & 0 & 1 \\
0 & 0 & 0 \\
1 & 1 & 0
\end{array}\right), \\
A_{d}=\left(\begin{array}{ccc}
0.1 & 0 & 0.1 \\
0 & 0.1 & 0 \\
0.1 & -0.2 & 0.1
\end{array}\right), \quad B_{d}=\left(\begin{array}{c}
0 \\
1 \\
-1
\end{array}\right), \quad C_{d}=\left(\begin{array}{ccc}
1 & -0.2155 & -0.0812 \\
0.0385 & 0.1 & -0.9713 \\
1.3851 & 0.8964 & 0.1
\end{array}\right), \\
D_{c}=0, \quad D_{d}=0 .
\end{gathered}
$$

The matrices appeared in (3.31) and (3.30) are given by $Q_{c}=4, S_{\mathrm{c}}=0, R_{c}=0.1 I_{3} ; Q_{d}=$ $4, S_{d}=0, R_{d}=-I_{3} ; T_{c}=10, T_{d}=1$ and

$$
P_{c}=\left(\begin{array}{lll}
7.6 & 3.8 & 3.8 \\
3.8 & 4.4 & 1.4 \\
3.8 & 1.4 & 2.4
\end{array}\right), \quad P_{d}=\left(\begin{array}{ccc}
2.96 & 1.01 & -0.04 \\
1.01 & 0.98 & 0.01 \\
-0.04 & 0.01 & 0.96
\end{array}\right)
$$

By solving (3.32), we obtain

$$
X=\left(\begin{array}{lll}
3.00 & 1.00 & 0.00 \\
1.00 & 1.00 & 0.00 \\
0.00 & 0.00 & 1.00
\end{array}\right)
$$

Thus, by Theorem 3.8, this system is dissipative under the quadratic supply rate. Moreover, we see that $X_{c}=4>0$ and $X_{d}=4>0$. And by solving (3.42), we get $a=0.169, b=0.32$, and

$$
K_{c}=\left(\begin{array}{lll}
0.0396 & 0.0198 & 0.0198
\end{array}\right), \quad K_{d}=\left(\begin{array}{lll}
0 & -0.1 & 0
\end{array}\right) .
$$

Thus, by (3.14) of Corollary 3.3, if $\Delta_{\text {sup }}$ satisfies $\Delta_{\text {sup }}<\ln b^{-1} / \ln (1+a)=7.291$, that is, $2 \leq \Delta_{\text {sup }} \leq 7$, then, all the conditions of Corollary 3.9 are satisfied. Therefore, $\left(u_{c}, u_{d}\right)=$ 
$\left(K_{c} x, K_{d} x\right)$ given by (4.4) can stabilize the closed-loop system and minimizes $J^{k_{f}}\left(x_{0}, u_{c}, u_{d}\right)$, that is, if $x_{0}=\left(\begin{array}{lll}0.1 & 1 & -0.5\end{array}\right)^{T}$, then

$$
J^{k_{f}}\left(x_{0}, K_{c} x, K_{d} x\right) \leq x_{0}^{T} X x_{0}=1.48=J^{\infty}\left(x_{0}, K_{c} x, K_{d} x\right)
$$

\section{Conclusions}

In this paper, by establishing the GUAS results for DIHS, we have obtained the conditions under which a dissipative DIHS with a hybrid performance functional can be asymptotically stabilized by a feedback control law and meantime the hybrid performance functional is optimized. For the case of linear DIHS with a quadratic supply rate and a quadratic hybrid performance functional, the corresponding sufficient conditions are changed into matrix inequalities. One example verifies the theoretic results obtained.

\section{Acknowledgments}

The authors would like to thank the Editor, Professor Jianshe S. Yu, and the anonymous referees for their helpful comments and suggestions. This work was supported by NSFCChina (no. 60874025) and ARC-Australia (no. DP0881391).

\section{References}

[1] J. C. Willems, "Dissipative dynamical systems—-part I: general theory," Archive for Rational Mechanics and Analysis, vol. 45, pp. 321-351, 1972.

[2] J. C. Willems, "Dissipative dynamical systems-part II: linear systems with quadratic supply rates," Archive for Rational Mechanics and Analysis, vol. 45, no. 5, pp. 352-393, 1972.

[3] D. Hill and P. Moylan, "The stability of nonlinear dissipative systems," IEEE Transactions on Automatic Control, vol. 21, no. 5, pp. 708-711, 1976.

[4] D. J. Hill and P. J. Moylan, "Dissipative dynamical systems: basic input-output and state properties," Journal of the Franklin Institute, vol. 309, no. 5, pp. 327-357, 1980.

[5] C. I. Byrnes and A. Isidori, "New results and examples in nonlinear feedback stabilization," Systems $\mathcal{E}$ Control Letters, vol. 12, no. 5, pp. 437-442, 1989.

[6] C. I. Byrnes, A. Isidori, and J. C. Willems, "Passivity, feedback equivalence, and the global stabilization of minimum phase nonlinear systems," IEEE Transactions on Automatic Control, vol. 36, no. 11, pp. 1228-1240, 1991.

[7] V. Lakshmikantham, D. D. Baĭnov, and P. S. Simeonov, Theory of Impulsive Differential Equations, vol. 6 of Series in Modern Applied Mathematics, World Scientific, Teaneck, NJ, USA, 1989.

[8] T. Yang, Impulsive Control Theory, vol. 272 of Lecture Notes in Control and Information Sciences, Springer, Berlin, Germany, 2001.

[9] Z. Li, Y. Soh, and C. Wen, Switched and Impulsive Systems: Analysis, Design and Application, vol. 313 of Lecture Notes in Control and Information Sciences, Springer, Berlin, Germany, 2005.

[10] Y. Zhang and J. Sun, "Stability of impulsive linear differential equations with time delay," IEEE Transactions on Circuits and Systems II, vol. 52, no. 10, pp. 701-705, 2005.

[11] Z. Guan, D. J. Hill, and X. Shen, "On hybrid impulsive and switching systems and application to nonlinear control," IEEE Transactions on Automatic Control, vol. 50, no. 7, pp. 1058-1062, 2005.

[12] M. V. Basin and M. A. Pinsky, "On impulse and continuous observation control design in Kalman filtering problem," Systems E Control Letters, vol. 36, no. 3, pp. 213-219, 1999.

[13] W.-H. Chen and W. X. Zheng, "Global exponential stability of impulsive neural networks with variable delay: an LMI approach," IEEE Transactions on Circuits and Systems I, vol. 56, no. 6, pp. 1248 $1259,2009$. 
[14] B. Liu, X. Liu, and K. L. Teo, "Feedback stabilization of dissipative impulsive dynamical systems," Information Sciences, vol. 177, no. 7, pp. 1663-1672, 2007.

[15] W.-H. Chen, J.-G. Wang, Y.-J. Tang, and X. Lu, “Robust $H_{\infty}$ control of uncertain linear impulsive stochastic systems," International Journal of Robust and Nonlinear Control, vol. 18, no. 13, pp. 1348-1371, 2008.

[16] W.-H. Chen and W. X. Zheng, "Robust stability and $H_{\infty}$-control of uncertain impulsive systems with time-delay," Automatica, vol. 45, no. 1, pp. 109-117, 2009.

[17] J. Zhao and D. J. Hill, "Dissipativity theory for switched systems," IEEE Transactions on Automatic Control, vol. 53, no. 4, pp. 941-953, 2008.

[18] W. M. Haddad, V. Chellaboina, and N. A. Kablar, "Nonlinear impulsive dynamical systems-part I: stability and dissipativity," in Proceedings of the 38th IEEE Conference on Decision and Control (CDC '99), vol. 5, pp. 4404-4422, Phoenix, Ariz, USA, December 1999.

[19] W. M. Haddad, V. Chellaboina, and N. A. Kablar, "Nonlinear impulsive dynamical systems-part II: feedback interconnections and optimality," in Proceeedings of the 38th IEEE Conference on Decision and Control (CDC'99), vol. 5, pp. 5225-5234, Phoenix, Ariz, USA, December 1999.

[20] W. M. Haddad, Q. Hui, V. Chellaboina, and S. Nersesov, "Vector dissipativity theory for discrete-time large-scale nonlinear dynamical systems," Advances in Difference Equations, vol. 2004, no. 1, pp. 37-66, 2004.

[21] W. M. Haddad, V. Chellaboina, and S. G. Nersesov, Impulsive and Hybrid Dynamical Systems: Stability, Dissipativity, and Control, Princeton Series in Applied Mathematics, Princeton University Press, Princeton, NJ, USA, 2006.

[22] W. M. Haddad and Q. Hui, "Dissipativity theory for discontinuous dynamical systems: basic input, state, and output properties, and finite-time stability of feedback interconnections," Nonlinear Analysis: Hybrid Systems, vol. 3, no. 4, pp. 551-564, 2009.

[23] B. Liu, X. Liu, and X. Liao, "Robust dissipativity for uncertain impulsive dynamical systems," Mathematical Problems in Engineering, vol. 2003, no. 3-4, pp. 119-128, 2003.

[24] M. Lei and B. Liu, "Robust impulsive synchronization of discrete dynamical networks," Advances in Difference Equations, vol. 2008, Article ID 184275, 17 pages, 2008.

[25] L. P. LaSalle and S. Lefschetz, Stability by Lyapunov's Direct Method, Academic Press, New York, NY, USA, 1961.

[26] B. Liu and D. J. Hill, "Comparison principle and stability of discrete-time impulsive hybrid systems," IEEE Transactions on Circuits and Systems I, vol. 56, no. 1, pp. 233-245, 2009.

[27] B. Liu and X. Liu, "Robust stability of uncertain discrete impulsive systems," IEEE Transactions on Circuits and Systems II, vol. 54, no. 5, pp. 455-459, 2007.

[28] B. Liu and X. Liu, "Uniform stability of discrete impulsive systems," International Journal of Systems Science, vol. 39, no. 2, pp. 181-192, 2008.

[29] V. Azhmyakov, V. G. Boltyanski, and A. Poznyak, "Optimal control of impulsive hybrid systems," Nonlinear Analysis: Hybrid Systems, vol. 2, no. 4, pp. 1089-1097, 2008.

[30] R. T. N. Cardoso and R. H. C. Takahashi, "Solving impulsive control problems by discrete-time dynamic optimization methods," Tendências em Matemática Aplicada e Computacional, vol. 9, no. 1, pp. 21-30, 2008.

[31] S. P. Boyd, L. El Ghaoui, E. Feron, and V. Balakrishnan, Linear Matrix Inequalities in System and Control Theory, SIAM, Philadelphia, Pa, USA, 1994. 